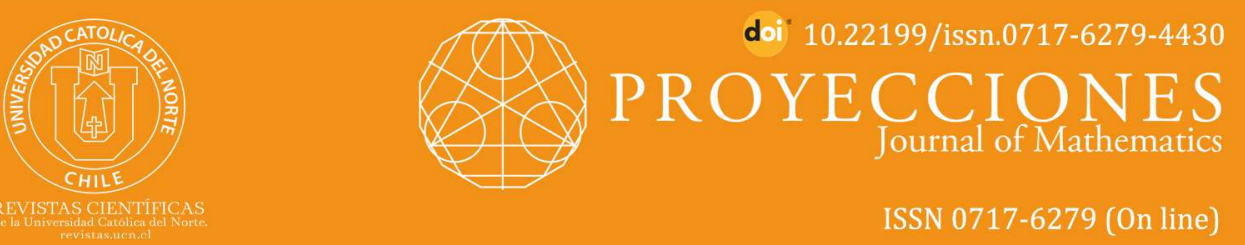

\title{
Basarab loop and the generators of its total multiplication group
}

\section{T. G. Jaiyéolá1 ${ }^{1}$ orcid.org/0000-0002-8695-5478}

G. O. Effiong ${ }^{2}$-10 orcid.org/0000-0001-9569-1905

${ }^{1}$ Obafemi Awolowo University, Dept of Mathematics. Ile-Ife, Nigeria.

-jaiyeolatemitope@yahoo.com

${ }^{2}$ Hezekiah University, Dept. of Computer Science and Mathematics. Umudi, Nkwerre, Nigeria.

videonoeffiong@gmail.com

Received: 2020/08/29 | Accepted: 2021/01/29

\begin{abstract}
:
A loop $(Q, \cdot)$ is called a Basarab loop if the identities: $\left(x \cdot y x^{\rho}\right)(x z)=x \cdot y z$ and $(y x) \cdot\left(x^{\lambda} z \cdot x\right)=y z \cdot x$ hold. It was shown that the left, right and middle nuclei of the Basarab loop coincide, and the nucleus of a Basarab loop is the set of elements $x$ whose middle inner mapping $T_{x}$ are automorphisms. The generators of the inner mapping group of a Basarab loop were refined in terms of one of the generators of the total inner mapping group of a Basarab loop. Necessary and sufficient condition(s) in terms of the inner mapping group (associators) for a loop to be a Basarab loop were established. It was discovered that in a Basarab loop: the mapping $x \mapsto T_{x}$ is an endomorphism if and only if the left (right) inner mapping is a left (right) regular mapping. It was established that a Basarab loop is a left and right automorphic loop and that the left and right inner mappings belong to its middle inner mapping group. A Basarab loop was shown to be an automorphic loop (A-loop) if and only if it is a middle automorphic loop (middle Aloop). Some interesting relations involving the generators of the total multiplication group and total inner mapping group of a Basarab loop were derived, and based on these, the generators of the total inner mapping group of a Basarab loop were finetuned. A Basarab loop was shown to be a totally automorphic loop (TA-loop) if and only if it is a commutative and flexible loop. These aforementioned results were used to give a partial answer to a 2013 question and an ostensible solution to a 2015 problem in the case of $\mathrm{Ba}$ sarab loop.
\end{abstract}

Keywords: Basarab loops; Inner mapping group; Automorphic loop (A-loop).

MSC (2020): 20N02, 20N05.

\section{Cite this article as (IEEE citation style):}

T. G. Jaiyéolá and G. O. Effiong, "Basarab loop and the generators of its total multiplication group", Proyecciones (Antofagasta, On line), vol. 40, no. 4, pp. 939-962, 2021, doi: 10.22199/issn.0717-6279-4430

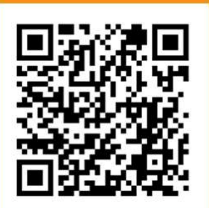

Article copyright: (C) 2021 T. G. Jaiyéọlá and G. O. Effiong. This is an open access article distributed under the terms of the Creative Commons License, which permits unrestricted use and distribution provided the original author and source are credited.

(cc) BY 


\section{Introduction}

Let $G$ be a non-empty set. Define a binary operation $(\cdot)$ on $G$. If $x \cdot y \in G$ for all $x, y \in G$, then the pair $(G, \cdot)$ is called a groupoid or Magma.

If each of the equations:

$$
a \cdot x=b \quad \text { and } \quad y \cdot a=b
$$

has unique solutions in $G$ for $x$ and $y$ respectively, then $(G, \cdot)$ is called a quasigroup.

If there exists a unique element $e \in G$ called the identity element such that for all $x \in G, x \cdot e=e \cdot x=x,(G, \cdot)$ is called a loop. We write $x y$ instead of $x \cdot y$, and stipulate that - has lower priority than juxtaposition among factors to be multiplied. For instance, $x \cdot y z$ stands for $x(y z)$.

Let $x$ be a fixed element in a groupoid $(G, \cdot)$. The left and right translation maps of $G, L_{x}$ and $R_{x}$ respectively are defined by

$$
y L_{x}=x \cdot y \quad \text { and } \quad y R_{x}=y \cdot x .
$$

It can now be seen that a groupoid $(G, \cdot)$ is a quasigroup if its left and right translation mappings are permutations. Since the left and right translation mappings of a quasigroup are bijective, then the inverse mappings $L_{x}^{-1}$ and $R_{x}^{-1}$ exist. Let

$$
x \backslash y=y L_{x}^{-1}=x M_{y} \quad \text { and } \quad x / y=x R_{y}^{-1}=y M_{x}^{-1}
$$

and note that

$$
x \backslash y=z \Leftrightarrow x \cdot z=y \quad \text { and } \quad x / y=z \Leftrightarrow z \cdot y=x .
$$

In a loop $(G, \cdot)$ with identity element $e$, the left inverse element of $x \in G$ is the element $x J_{\lambda}=x^{\lambda} \in G$ such that

$$
x^{\lambda} \cdot x=e
$$

while the right inverse element of $x \in G$ is the element $x J_{\rho}=x^{\rho} \in G$ such that

$$
x \cdot x^{\rho}=e .
$$

If $x^{\lambda}=x^{\rho}$ for any $x \in G$, then we simply write $x^{\lambda}=x^{\rho}=x^{-1}$ or $J_{\lambda}=J_{\rho}=J$. Let $a, b$ and $c$ be three elements of a loop $G$. The loop 
associator of $a, b$ and $c$ is the unique element $(a, b, c)$ of $G$ which satisfies $(a b) c=\{a(b c)\}(a, b, c)$. The loop commutator of $a$ and $b$ is the unique element $(a, b)$ of $G$ which satisfies $(a b)=(b a)(a, b)$.

The right nucleus of $G$ is defined by $N_{\rho}(G, \cdot)=\{a \in G \mid z y \cdot a=$ $z \cdot y a \forall y, z \in G\}$. The left nucleus of $G$ is defined by $N_{\lambda}(G, \cdot)=\{a \in$ $G: a x \cdot y=a \cdot x y \forall x, y \in G\}$. The middle nucleus of $G$ is defined by $N_{\mu}(G, \cdot)=\{a \in G: y a \cdot x=y \cdot a x \forall x, y \in G\}$. The nucleus of $G$ is defined by $N(G, \cdot)=N_{\lambda}(G, \cdot) \cap N_{\rho}(G, \cdot) \cap N_{\mu}(G, \cdot)$. The centrum of $G$ is defined by $C(G, \cdot)=\{a \in G: a x=x a \forall x \in G\}$. The center of $G$ is defined by $Z(G, \cdot)=N(G, \cdot) \cap C(G, \cdot) . N_{\rho}(G, \cdot), N_{\lambda}(G, \cdot), N_{\mu}(G, \cdot), N(G, \cdot), Z(G, \cdot)$ are subgroups of $(G, \cdot)$.

The group of all permutations on $G$ is called the permutation group of $G$ and denoted by $S Y M(G)$. The group $\mathcal{M}(G, \cdot)=\left\langle\left\{R_{x}, L_{x},: x \in G\right\}\right\rangle$ is called the multiplication group of $(G, \cdot)$ and $\mathcal{M}(G, \cdot) \leq S Y M(G)$.

If $e \alpha=e$ in a loop $G$ such that $\alpha \in \mathcal{M}(G)$, then $\alpha$ is called an inner mapping and they form a group $\operatorname{Inn}(G)$ called the inner mapping group. The right, left and middle inner mappings

$$
R_{(x, y)}=R_{x} R_{y} R_{x y}^{-1}, L_{(x, y)}=L_{x} L_{y} L_{y x}^{-1} \text { and } T_{x}=R_{x} L_{x}^{-1}
$$

respectively generate the right inner mapping group $\operatorname{Inn}_{\rho}(G)$, left inner mapping group $\operatorname{Inn}_{\lambda}(G)$ and the middle inner mapping group $\operatorname{Inn}_{\mu}(G)$. if

The triple $(A, B, C)$ of bijections of a loop $(G, \cdot)$ is called an autotopism

$$
x A \cdot y B=(x \cdot y) C \forall x, y \in G .
$$

Such triples form a group $A U T(G, \cdot)$ called the autotopism group of $(G, \cdot)$. Furthermore, if $A=B=C$, then $A$ is called an automorphism of $(G, \cdot)$. Such bijections form a group $\operatorname{AUM}(G, \cdot)$ called the automorphism group of $(G, \cdot)$. If

$$
\operatorname{Inn}_{\lambda}(G) \leq A U M(G), \operatorname{Inn}_{\rho}(G) \leq A U M(G), \text { and } \operatorname{Inn}_{\mu}(G) \leq A U M(G),
$$

then $G$ is called a left $\mathrm{A}$-loop $\left(\mathrm{A}_{\lambda}\right.$-loop$)$, right $\mathrm{A}$-loop( $\mathrm{A}_{\rho}$-loop $)$ and middle A-loop $\left(\mathrm{A}_{\mu}\right.$-loop $)$ respectively. It is well known that

$\operatorname{Inn}(G, \cdot)=\left\langle R_{(x, y)}, L_{(x, y)}, T_{x} \mid x, y \in G\right\rangle$, but this was later improved 
to be $\operatorname{Inn}(G, \cdot)=\left\langle L_{(x, y)}, T_{x} \mid x, y \in G\right\rangle=\left\langle R_{(x, y)}, T_{x} \mid x, y \in G\right\rangle$ in Vojtěchovský [32].

If $\operatorname{Inn}(G, \cdot) \leq \operatorname{AUM}(G, \cdot)$, then $(G, \cdot)$ is called an automorphic loop (A-loop)

The group $\mathcal{T} \mathcal{M}(G, \cdot)=\left\langle\left\{R_{x}, L_{x}, M_{x}: x \in G\right\}\right\rangle$ is called the total multiplication group of $(G, \cdot)$ and an element $\alpha \in \mathcal{T} \mathcal{M}(G, \cdot)$ such that $e \alpha=e$ is called a total inner mapping of $(G, \cdot)$. It has been established in Stanovský et al. [25] that the total inner mapping group

$$
\begin{gathered}
\operatorname{TInn}(G, \cdot)=\left\langle R_{(x, y)}, L_{(x, y)}, T_{x}, M_{(x, y)}, U_{x} \mid x, y \in G\right\rangle, \text { where } \\
M_{(x, y)}=M_{y} M_{x} M_{y \backslash x}^{-1}
\end{gathered}
$$

and $U_{x}=M_{x} R_{x}^{-1}$. Although, Syrbu [28] showed that

$$
\begin{gathered}
\operatorname{TInn}(G, \cdot)=\left\langle R_{(x, y)}, L_{(x, y)}, T_{x}, P_{(x, y)}, P_{(x, y)}^{\prime}, U_{x}^{-1}, V_{x} \mid x, y \in G\right\rangle \text {, where } \\
P_{(x, y)}=M_{x} M_{y} L_{x} R_{y}^{-1},
\end{gathered}
$$

$P_{(x, y)}^{\prime}=M_{y}^{-1} M_{x}^{-1} R_{y} L_{x}^{-1}$ and $V_{x}=R_{x} M_{x}$ but this result was later on improved to $\operatorname{TInn}(G, \cdot)=\left\langle R_{(x, y)}, L_{(x, y)}, T_{x}, P_{(x, y)}, V_{x} \mid x, y \in G\right\rangle$ by Syrbu and Grecu [30]. In an inverse property loop $(G, \cdot)$,

$\operatorname{TM}(\mathrm{G}, \cdot)=\left\langle\left\{L_{x}, J: x \in G\right\}\right\rangle=<\left\{M_{x}: x \in G\right\}>$ and $\operatorname{TInn}(G, \cdot)=\langle\operatorname{Inn}(G, \cdot), J \mid x, y \in G\rangle=\left\langle M_{(x, y)} \mid x, y \in G\right\rangle$.

If $\operatorname{TInn}(G, \cdot) \leq A U M(G, \cdot)$, then $(G, \cdot)$ is called a totally automorphic loop (TA-loop). TA-loops are commutative. Some works in the direction of structure of A-loops and TA-loops can be found in $[6,16,18,19,20$, $22,25,32]$. According to Stanovský et al. [25], $\operatorname{TInn}(G, \cdot)$ characterizes normal subloops.

Theorem 1.1. (Kinyon [18]) A loop is a TA-loop if and only if it is a commutative Moufang loop.

The above fact is gotten from the result below.

Theorem 1.2. (Kinyon [18]) Let $(G, \cdot)$ be a loop. The following are equivalent: 
1. $\left\langle L_{x \backslash y}^{-1} M_{y} M_{x}, R_{y / x}^{-1} M_{y}^{-1} M_{x}^{-1} \mid x, y \in G\right\rangle \leq A U M(G, \cdot)$.

2. $(G, \cdot)$ is both an A-loop and a Moufang loop.

And Theorem 1.2 led to the following Question 1.1.

Question 1.1. (Kinyon [18]) What other interesting varieties of loops can be characterized by specifying that some group of total inner mappings acts as automorphisms?

Question 1.2. (Stanovský et al. [25]) Are the generating sets of TInn $(G)$ for a loop $G$ minimal, i.e, can any of the five types of mappings be removed? Is there a generating set for $\mathcal{T} \mathcal{M}(Q)$ with only two types of inner mappings?

Remark 1.1. Syrbu and Grecu [30] were able to provide answers to Question 1.2 by showing that:

1. If $Q$ is a power associative loop, then

$$
\operatorname{TInn}(Q)=\left\langle R_{(x, y)}, L_{(x, y)}, P_{(x, y)}, V_{x} \mid x, y \in Q\right\rangle .
$$

2. If $Q$ is a middle Bol loop, then

$$
\operatorname{TInn}(Q)=\left\langle R_{(x, y)}, P_{(x, y)}, V_{x} \mid x, y \in Q\right\rangle .
$$

The authors furthermore established the importance of the total inner mapping group by showing that if $Q$ is a middle Bol loop, then $\operatorname{Inn}(Q) \triangleleft \operatorname{TInn}(Q)$. But this result is not necessarily true for a left or right Bol loop (note that, every middle Bol loop corresponds to a right or left Bol loop and vice versa). It must be recalled that Grecu and Syrbu [13], Syrbu [27], Syrbu and Grecu [29] established isostrophy invariance in several structural properties between middle Bol loops and their corresponding right or left Bol loop.

For an overview of the theory of loops, readers may check $[7,8,9,12$, $15,21,23,31]$.

Definition 1.1. Let $(G, \cdot)$ be a quasigroup. Then

1. $U \in S Y M(G)$ is called $\lambda$-regular if there exists $(U, I, U) \in \operatorname{AUT}(G, \cdot)$; the set of all such mappings forms a group $\Lambda(G, \cdot)$ called the group of left regular mappings. 
2. a bijection $U$ is called $\rho$-regular if there exists $(I, U, U) \in A U T(G, \cdot)$; the set of all such mappings forms a group $\mathcal{P}(G, \cdot)$ called the group of right regular mappings.

Definition 1.2. A loop $(G, \cdot)$ is called a cross inverse property loop $(C I P L)$ if it obeys the identity $\mathrm{xy} \cdot x^{\rho}=y \quad$ or $\quad x \cdot y x^{\rho}=y \quad$ or $\quad x^{\lambda} \cdot(y x)=$ $y \quad$ or $\quad x^{\lambda} y \cdot x=y$ for all $x, y, \in G$.

A loop $(G, \cdot)$ is called an automorphic inverse property loop(AIPL) if it obeys the identity

$(\mathrm{xy})^{\rho}=x^{\rho} y^{\rho} \quad$ or $\quad(x y)^{\lambda}=x^{\lambda} y^{\lambda}$ for all $x, y, \in G$.

A loop satisfying the identical relation $\mathrm{xy} \cdot z x=(x \cdot y z) x$ is called a Moufang loop.

A loop $(Q, \cdot)$ is called a Basarab loop (or $K$-loop), if the identities:

$$
\underbrace{\left(x \cdot y x^{\rho}\right) \cdot x z=x \cdot y z}_{B K 1}, \quad \underbrace{y x \cdot\left(x^{\lambda} z \cdot x\right)=y z \cdot x}_{B K 2}
$$

hold for all $x, y, z \in Q$.

Example 1.1. (Basarab [4])

Let $\mathcal{F}$ be a field, $\mathcal{F}^{\prime}$ be the set of non-zero elements of $\mathcal{F}$. Define on the set $\mathcal{Q}=\mathcal{F}^{\prime} \times \mathcal{F}$ the operation $(\cdot)$ as follows:

$$
(a, x) \cdot(b, y)=\left(a \cdot b,\left(a^{-1}-1\right) \cdot\left(b^{-1}-1\right)+b^{-1} x+y\right) .
$$

Then, $(\mathcal{Q}, \cdot)$ is a Basarab loop.

In recent years, many works have been published with the name Kloop(also called Bruck-loop, Bol-Bruck-loop). Kerby and Wefelscheid investigated the additive structure of a near-domain with extra axioms and then they called the new structure a K-loop, but according to Kiechle [14], they used the term K-loop only in talks in 1970s and the beginning of 1980s. Ungar [26] and Kiechle [14] continued to study the K-loop introduced by Kerby and Wefelscheid.

On the other hand, the term 'K-loop' was used for different purposes by Soikis [24] in 1970, and latter but independently by Basarab [2] in 1992. Thus, the notion of 'K-loop' as used by Soikis, Ungar and Basarab has different meaning respectively, and may be confusing. For example, the 
book titled theory of k-loops written by Kiechle [14] has completely different meaning from the paper titled K-loops published by Basarab [2].

Basarab loops (also called K-loops) are non-associative generalizations of groups. In this paper, we shall adopt the name 'Basarab loop', to refer to 'K-loop' of Basarab, as recommended by R.Artzy in the review of Basarab [4].

The first publications introducing the class of loop called Basarab loop are the two prominent papers of Basarab [1,2] in 1992. Basarab [4] used the result published by Belousov [5] to construct an example of a Basarab loop whose nucleus is an abelian group. After his first publications in 1992 on Basarab loops, no other author researched on properties of Basarab loops until in 1996 and 1997, that he, Basarab studied the relationship between a generalized Moufang loop, Osborn loop, VD-loop, and a Basarab loop, and a special type of a Basarab loop, known as IK-loop respectively. In Jaiyéolá and Effiong [17], the authors considered the Basarab loop and its invariance with inverse properties. Effiong et al. [10, 11] respectively investigated the connections between Basarab loop and Buchsteiner loop, and the holomorphy of Basarab loop. In this current study, an IK-loop is an automorphic inverse property Basarab loop.

Here are some existing results on Basarab loop.

Theorem 1.3. (Basarab [4]) Let $(Q, \cdot)$ be a Basarab loop.

1. $N(Q, \cdot)$ contains the associator of any three elements of $Q$.

2. The quotient loop $Q / Z(Q, \cdot)$ is a group.

3. If $(Q, \cdot)$ is generated by one element, then it is solvable.

4. If $(Q, \cdot)$ has the automorphic inverse property, then it is nilpotent.

Theorem 1.4. (Basarab [2]) Let $(Q, \cdot)$ be a Basarab loop.

1. $N(Q, \cdot)$ is a nontrivial normal subloop.

2. The quotient loop $Q / N(Q, \cdot)$ is an abelian.

3. If $N(Q, \cdot)$ has an odd order, then $(Q, \cdot)$ is solvable.

Theorem 1.5. (Basarab [3])

1. Any Basarab loop (any VD-loop) is a G-loop. 
2. Any Basarab loop (VD-loop) is an Osborn loop.

3. A Basarab loop $(Q, \cdot)$ is a $V D$-loop if $x^{2} \in N(Q, \cdot)$ for any $x \in Q$.

4. A VD-loop $(Q, \cdot)$ is a Basarab loop if $x^{2} \in N(Q, \cdot)$ for any $x \in Q$.

In this present work, the nuclei of Basarab loops are characterized in terms of middle inner mappings, associators in Basarab loops are expressed in terms of total inner mappings and necessary and sufficient condition on generator(s) of the inner mapping group is given in order for a Basarab loop to be an A-loop. Some results on the generators of the inner mapping and total inner mapping groups of a Basarab loop are established in order to show that a class of total inner mappings act on a Basarab loop $Q$ by automorphisms if and only if $Q$ is an A-loop and flexible.

\section{Main Results}

\subsection{Some Algebraic properties of Basarab loop}

Lemma 2.1. Let $(Q, \cdot)$ be a Basarab loop, then the following hold for all $x, y, z \in Q$ :

(i) $\left(x \cdot y x^{\rho}\right) x=x y$.

(ii) $\left(x \cdot y x^{\rho}\right) \cdot x y^{\rho}=x$.

(iii) $\left(x \cdot z^{\lambda} x^{\rho}\right) \cdot x z=x$.

(iv) $\left(z^{\lambda} x\right)\left(x^{\lambda} z \cdot x\right)=x$.

(v) $x\left(x^{\lambda} z \cdot x\right)=z x$.

(vi) $y x \cdot\left(x^{\lambda} y^{\rho} \cdot x\right)=x$.

(vii) $T_{x}^{-1}=R_{x^{\rho}} L_{x}$.

(viii) $T_{x}=L_{x^{\lambda}} R_{x}$.

(ix) $\left[L_{x^{\lambda}} R_{x}, R_{x^{\rho}} L_{x}\right]=I$.

(x) $x \cdot\left(x^{\lambda} y \cdot x\right) x^{\rho}=y$.

(xi) $x^{\lambda}\left(x \cdot y x^{\rho}\right) \cdot x=y$ 
Proof. The proof of (i) to (vi) are gotten from (1.2) by making the following substitutions: $z=e, z=y^{\rho}, y=z^{\lambda}$ in BK1 and $y=z^{\lambda}, y=$ $e, z=y^{\rho}$ in BK2.

(vii) follows from (i): $R_{x^{\rho}} L_{x} R_{x}=L_{x} \Leftrightarrow R_{x^{\rho}} L_{x}=L_{x} R_{x}^{-1} \Leftrightarrow T_{x}^{-1}=R_{x^{\rho}} L_{x}$. (viii) follows from (v): $L_{x^{\lambda}} R_{x} L_{x}=R_{x} \Leftrightarrow L_{x^{\lambda}} R_{x}=R_{x} L_{x}^{-1} \Leftrightarrow T_{x}=$ $L_{x^{\lambda}} R_{x}$. (ix) to (xi): From (i), $R_{x^{\rho}} L_{x} R_{x}=L_{x} \Rightarrow R_{x^{\rho}} L_{x}=L_{x} R_{x}^{-1}=$ $\left(R_{x} L_{x}^{-1}\right)^{-1}=T_{x}^{-1}$. From (v), $L_{x^{\lambda}} R_{x} L_{x}=R_{x} \Rightarrow L_{x^{\lambda}} R_{x}=R_{x} L_{x}^{-1}=$ $T_{x}$. Thus, $\quad T_{x} T_{x}^{-1}=L_{x^{\lambda}} R_{x} R_{x^{\rho}} L_{x}=I=T_{x}^{-1} T_{x}=R_{x^{\rho}} L_{x} L_{x^{\lambda}} R_{x} \Rightarrow$ $L_{x^{\lambda}} R_{x} R_{x^{\rho}} L_{x}=R_{x^{\rho}} L_{x} L_{x^{\lambda}} R_{x}$. Hence, $\left[L_{x^{\lambda}} R_{x}, R_{x^{\rho}} L_{x}\right]=I$ and $x \cdot\left(x^{\lambda} y\right.$. $x) x^{\rho}=y=x^{\lambda}\left(x \cdot y x^{\rho}\right) \cdot x$ for all $x \in Q$.

Lemma 2.2. Let $(Q, \cdot)$ be a loop. $(Q, \cdot)$ is a Basarab loop if and only if $\left(R_{x^{\rho}} L_{x}, L_{x}, L_{x}\right),\left(R_{x}, L_{x^{\lambda}} R_{x}, R_{x}\right) \in \operatorname{AUT}(Q, \cdot)$ if and only if $\left(T_{x}^{-1}, L_{x}, L_{x}\right),\left(R_{x}, T_{x}, R_{x}\right) \in \operatorname{AUT}(Q, \cdot)$.

Proof. Simply put BK1 and BK2 of (1.2) in autotopic forms. The last part follows by (vii) and (viii) of Lemma 2.1.

\subsection{Nuclei of a Basarab loop}

Theorem 2.1. Let $(Q, \cdot)$ be a Basarab loop.

1. The following are equivalent:

(a) $x \in N_{\rho}(Q, \cdot)$ for all $x \in Q$.

(b) $x \in N_{\lambda}(Q, \cdot)$ for all $x \in Q$.

(c) $x \in N_{\mu}(Q, \cdot)$ for all $x \in Q$.

(d) $(y x)\left(x^{\lambda} z\right)=y z$ for all $x, y, z \in Q$.

(e) $\left(y x^{\rho}\right)(x z)=y z$ for all $x, y, z \in Q$.

(f) $T_{x} \in A U M(Q, \cdot)$ for all $x \in Q$.

(g) $\operatorname{Inn}_{\mu}(Q, \cdot) \leq A U M(Q, \cdot)$.

(h) $(Q, \cdot)$ is an $A_{\mu}$-loop.

(i) $(Q, \cdot)$ is a group.

2. $N(Q, \cdot)=N_{\lambda}(Q, \cdot)=N_{\rho}(Q, \cdot)=N_{\mu}(Q, \cdot)$.

3. $N(Q, \cdot)=\left\{x \in Q \mid T_{x} \in \operatorname{AUM}(Q, \cdot)\right\}$. 


\section{Proof.}

1. Let $(Q, \cdot)$ be a Basarab loop. Then

$$
\begin{aligned}
& x \in N_{\rho}(Q, \cdot) \Leftrightarrow\left(I, R_{x}, R_{x}\right) \in \operatorname{AUT}(Q, \cdot) \Leftrightarrow\left(I, R_{x}^{-1}, R_{x}^{-1}\right) \in \operatorname{AUT}(Q, \cdot) \\
& \Leftrightarrow\left(T_{x}^{-1}, L_{x}, L_{x}\right) \cdot\left(I, R_{x}^{-1}, R_{x}^{-1}\right) \in \operatorname{AUT}(Q, \cdot) \Leftrightarrow\left(T_{x}^{-1}, L_{x} R_{x}^{-1}, L_{x} R_{x}^{-1}\right) \\
& \in \operatorname{AUT}(Q, \cdot) \\
& \Rightarrow\left(T_{x}^{-1}, T_{x}^{-1}, T_{x}^{-1}\right) \in \operatorname{AUT}(Q, \cdot) \Leftrightarrow T_{x}^{-1} \in \operatorname{AUT}(Q, \cdot) \Leftrightarrow T_{x} \in \operatorname{AUM}(Q, \cdot) .
\end{aligned}
$$

Therefore, in a Basarab loop, $x \in N_{\rho}(Q, \cdot)$ if and only if $T_{x}$ is an automorphism of $(Q, \cdot)$.

Let $(Q, \cdot)$ be a Basarab loop. Then

$$
\begin{gathered}
\left(L_{x}^{-1}, I, L_{x}^{-1}\right) \in \operatorname{AUT}(Q, \cdot) \Leftrightarrow\left(L_{x}, I, L_{x}\right) \in \operatorname{AUT}(Q, \cdot) \Leftrightarrow x \in N_{\lambda}(Q, \cdot) \\
\Leftrightarrow\left(L_{x}, I, L_{x}\right) \in \operatorname{AUT}(Q, \cdot) \Leftrightarrow\left(L_{x}^{-1}, I, L_{x}^{-1}\right) \in \operatorname{AUT}(Q, \cdot) \\
\Leftrightarrow\left(R_{x}, T_{x}, R_{x}\right) \cdot\left(L_{x}^{-1}, I, L_{x}^{-1}\right) \in \operatorname{AUT}(Q, \cdot) \Leftrightarrow\left(R_{x} L_{x}^{-1}, T_{x}, R_{x} L_{x}^{-1}\right) \\
\in \operatorname{AUT}(Q, \cdot)\left(T_{x}, T_{x}, T_{x}\right) \in \operatorname{AUT}(Q, \cdot) \Leftrightarrow T_{x} \in \operatorname{AUM}(Q, \cdot) .
\end{gathered}
$$

Thus, in a Basarab loop $(Q, \cdot), x \in N_{\lambda}(Q, \cdot)$ if and only if $T_{x}$ is an automorphism of $(Q, \cdot)$.

Let $(Q, \cdot)$ be a Basarab loop. Then $x \in N_{\mu}(Q, \cdot) \Leftrightarrow\left(R_{x}, L_{x}^{-1}, I\right) \in$ $\operatorname{AUT}(Q, \cdot) \Leftrightarrow\left(R_{x}^{-1}, L_{x}, I\right) \in \operatorname{AUT}(Q, \cdot) \Leftrightarrow\left(R_{x}, T_{x}, R_{x}\right)\left(R_{x}^{-1}, L_{x}, I\right) \in$ $\operatorname{AUT}(Q, \cdot) \Leftrightarrow\left(R_{x} R_{x}^{-1}, T_{x} L_{x}, R_{x}\right) \Leftrightarrow\left(I, R_{x}, R_{x}\right) \in \operatorname{AUT}(Q, \cdot) \Leftrightarrow x \in$ $N_{\rho}(Q, \cdot)$. Thus, in a Basarab loop $(Q, \cdot), x \in N_{\mu}(Q, \cdot)$ if and only if $x \in N_{\rho}(Q, \cdot) . x \in N_{\lambda}(Q, \cdot) \Leftrightarrow\left(L_{x}, I, L_{x}\right) \in \operatorname{AUT}(Q, \cdot) \Leftrightarrow\left(L_{x}^{-1}, I, L_{x}^{-1}\right) \in$ $\operatorname{AUT}(Q, \cdot) \Leftrightarrow\left(R_{x^{\rho}} L_{x}, L_{x}, L_{x}\right) \cdot\left(L_{x}^{-1}, I, L_{x}^{-1}\right) \in \operatorname{AUT}(Q, \cdot) \Leftrightarrow\left(R_{x^{\rho}}, L_{x}, I\right) \in$ $\operatorname{AUT}(Q, \cdot) \Leftrightarrow(y x)\left(x^{\lambda} z\right)=y z \Leftrightarrow\left(y x^{\rho}\right)(x z)=y z$.

2. In a Basarab loop $(Q, \cdot), x \in N_{\lambda}(Q, \cdot)$ if and only if $T_{x}$ is an automorphism of $(Q, \cdot)$. Also, $x \in N_{\rho}(Q, \cdot)$ if and only if $T_{x}$ is an automorphism of $(Q, \cdot)$. This means $x \in N_{\lambda}(Q, \cdot) \subset N_{\rho}(Q, \cdot)$ if and only if $T_{x}$ is an automorphism of $(Q, \cdot)$. And $x \in N_{\rho}(Q, \cdot) \subset N_{\lambda}(Q, \cdot)$ if and only if $T_{x}$ is an automorphism of $(Q, \cdot)$. Hence, $N_{\lambda}(Q, \cdot)=N_{\rho}(Q, \cdot)$.

$$
\begin{gathered}
x \in N_{\mu}(Q, \cdot) \Leftrightarrow\left(R_{x}, L_{x}^{-1}, I\right) \in \operatorname{AUT}(Q, \cdot) \Leftrightarrow \\
\left(L_{x} R_{x}^{-1}, L_{x}, L_{x}\right)\left(R_{x}, L_{x}^{-1}, I\right) \in \operatorname{AUT}(Q, \cdot) \Leftrightarrow\left(L_{x}, I, L_{x}\right) \in \operatorname{AUT}(Q, \cdot) \\
\Leftrightarrow x \in N_{\lambda}(Q, \cdot) .
\end{gathered}
$$

Hence, $N_{\lambda}(Q, \cdot)=N_{\mu}(Q, \cdot)$.

3. Use 1. 


\subsection{Inner mappings and associators of Basarab loop}

Theorem 2.2. In any loop $(Q, \cdot)$, for all $x, y, z \in Q$ :

1. $z L_{(x, y)}=(y x) M_{(y \cdot x z)}$.

2. $(y, x, z)=\left[y x \cdot z L_{(x, y)}\right] M_{(y x \cdot z)}$.

3. $z R_{(x, y)}=(x y) M_{(z x \cdot y)}^{-1}$.

4. $(z, x, y)=(z \cdot y x) M_{\left[z R_{(x, y)} \cdot x y\right]}$.

5. $y T_{x}=x M_{(y x)}$.

6. $(y, x)=(x y) M_{\left(x \cdot y T_{x}\right)}$.

\section{Proof.}

1. $z L_{(x, y)}=z L_{x} L_{y} L_{y x}^{-1}=(y \cdot x z) L_{y x}^{-1}=(y x) M_{(y \cdot x z)}$.

2. Recall that $y x \cdot z=(y \cdot x z)(y, x, z) \Rightarrow y \cdot x z=(y x \cdot z) /(y, x, z)$. So, from $1, z L_{(x, y)}=(y \cdot x z) L_{y x}^{-1} \Rightarrow y \cdot x z=y x \cdot z L_{(x, y)} \Rightarrow(y x$. $z) /(y, x, z)=y x \cdot z L_{(x, y)} \Rightarrow(y, x, z) M_{(y x \cdot z)}^{-1}=y x \cdot z L_{(x, y)} \Rightarrow(y, x, z)=$ $\left[y x \cdot z L_{(x, y)}\right] M_{(y x \cdot z)}$.

3. $z R_{(x, y)}=z R_{x} R_{y} R_{x y}^{-1}=(z x \cdot y) R_{x y}^{-1}=(x y) M_{(z x \cdot y)}^{-1}$.

4. Recall that $z x \cdot y=(z \cdot x y)(z, x, y)$. So, from $3, z R_{(x, y)}=(z x \cdot y) R_{x y}^{-1} \Rightarrow$ $z x \cdot y=z R_{(x, y)} \cdot x y \Rightarrow(z \cdot x y)(z, x, y)=z R_{(x, y)} \cdot x y \Rightarrow(z, x, y)=$ $\left[z R_{(x, y)} \cdot x y\right] L_{(z \cdot x y)}^{-1} \Rightarrow(z, x, y)=(z \cdot y x) M_{\left[z R_{(x, y)} \cdot x y\right]}$.

5. $y T_{x}=y R_{x} L_{x}^{-1}=(y x) L_{x}^{-1}=x M_{(y x)}$.

6. Recall that $y x=(x y)(y, x)$. So, from $5, y T_{x}=[(x y)(y, x)] L_{x}^{-1} \Rightarrow$ $(x y)(y, x)=x \cdot y T_{x} \Rightarrow(y, x)=\left[x \cdot y T_{x}\right] L_{(x y)}^{-1}=(x y) M_{\left[x \cdot y T_{x}\right]} \Rightarrow$ $(y, x)=(x y) M_{\left[x \cdot y T_{x}\right]}$.

Theorem 2.3. Let $(Q, \cdot)$ be a loop. The following are equivalent: 
1. $(Q, \cdot)$ is a Basarab loop.

2. $(x, y, z)=\left[\left(x \cdot y x^{\rho}\right)(x z)\right] \backslash(x y \cdot z)$ and $(x, y, z)=(x \cdot y z) \backslash\left[(x z)\left(z^{\lambda} y \cdot z\right)\right]$ for all $x, y, z \in Q$

3. $\operatorname{Inn}(Q, \cdot)=\left\langle L_{x} L_{x \cdot y x^{\rho}} L_{x y}^{-1}, T_{x} \mid x, y \in Q\right\rangle$ and $\operatorname{Inn}(Q, \cdot)=\left\langle R_{x} R_{x^{\lambda} z \cdot x} R_{z x}^{-1}, T_{x} \mid x, z \in Q\right\rangle$.

\section{Proof.}

$\mathbf{1} \Leftrightarrow \mathbf{2}$ This is achieved by simply using (1.2) and the fact that $x y \cdot z=$ $(x \cdot y z)(x, y, z)$.

$\mathbf{1} \Leftrightarrow \mathbf{3} \mathrm{BK} 1$ of (1.2) is true if and only if $L_{y} L_{x}=L_{x} L_{x \cdot y x^{\rho}} \Leftrightarrow L_{y} L_{x} L_{x y}^{-1}=$ $L_{x} L_{x \cdot y x^{\rho}} L_{x y}^{-1} \Leftrightarrow L_{(y, x)}=L_{x} L_{x \cdot y x^{\rho}} L_{x y}^{-1} \Leftrightarrow \operatorname{Inn}(Q, \cdot)$

$=\left\langle L_{x} L_{x \cdot y x^{\rho}} L_{x y}^{-1}, T_{x} \mid x, y \in Q\right\rangle$. BK2 of (1.2) is true if and only if $R_{z} R_{x}=R_{x} R_{\left(x^{\lambda} z \cdot x\right)} \Leftrightarrow R_{z} R_{x} R_{z x}^{-1}=R_{x} R_{\left(x^{\lambda} z \cdot x\right)} R_{z x}^{-1} \Leftrightarrow R_{(z, x)}=$ $R_{x} R_{\left(x^{\lambda} z \cdot x\right)} R_{z x}^{-1} \Leftrightarrow \operatorname{Inn}(Q, \cdot)=\left\langle R_{x} R_{x^{\lambda} z \cdot x} R_{z x}^{-1}, T_{x} \mid x, z \in Q\right\rangle$.

Theorem 2.4. In a Basarab loop $(Q, \cdot)$, for all $x, y, z \in Q$ :

1. $(y, x, z)=\left[z L_{y x} J_{\lambda} \cdot z L_{(x, y)} L_{y x}\right] J_{\lambda}$.

2. $(z, x, y)=(z \cdot y x)^{\lambda}\left[z R_{(x, y)} \cdot x y\right]$.

3. $(x, y, z)=\left[y T_{x}^{-1} \cdot x z\right]^{\lambda}(x y \cdot z)$.

4. $(x, y, z)=(x \cdot y z)^{\lambda} \cdot(x z)\left(y T_{z}\right)$.

Proof. Note that in a Basarab loop $(Q, \cdot),(x, y, z) \in N(Q, \cdot)$.

1. From the proof of 2 of Theorem 2.2, $(y x \cdot z) /(y, x, z)=y x \cdot z L_{(x, y)} \Rightarrow$ $(y x \cdot z)(y, x, z)^{-1}=y x \cdot z L_{(x, y)} \Rightarrow(y, x, z)^{-1}=(y x \cdot z)^{\lambda}\left[y x \cdot z L_{(x, y)}\right] \Rightarrow$ $(y, x, z)=\left[z L_{y x} J_{\lambda} \cdot z L_{(x, y)} L_{y x}\right] J_{\lambda}$.

2. From the proof of 4 of Theorem $2.2,(z \cdot x y)(z, x, y)=z R_{(x, y)} \cdot x y \Rightarrow$ $(z, x, y)=(z \cdot y x)^{\lambda}\left[z R_{(x, y)} \cdot x y\right]$. 
3. By 2 of Theorem 2.3, xy $z=x(y z) \cdot(x, y, z) \Rightarrow x y \cdot z=\left(x \cdot y x^{\rho}\right)(x z)$. $(x, y, z) \Rightarrow\left[\left(x \cdot y x^{\rho}\right)(x z)\right]^{\lambda}(x y \cdot z)=(x, y, z) \Rightarrow\left[y T_{x}^{-1} \cdot x z\right]^{\lambda}(x y \cdot z)=$ $(x, y, z)$.

4. By 2 of Theorem 2.3, xy $z=x(y z) \cdot(x, y, z) \Rightarrow(x \cdot y z)(x, y, z)=$ $(x z)\left(z^{\lambda} y \cdot z\right) \Rightarrow(x, y, z)=(x \cdot y z)^{\lambda} \cdot(x z)\left(z^{\lambda} y \cdot z\right) \Rightarrow(x, y, z)=$ $(x \cdot y z)^{\lambda} \cdot(x z)\left(y T_{z}\right)$.

Corollary 2.1. In a Basarab loop $(Q, \cdot)$ :

1. $\operatorname{Inn}(Q, \cdot)=\left\langle L_{x} L_{y T_{x}^{-1}} L_{x y}^{-1}, T_{x} \mid x, y \in Q\right\rangle$.

2. $\operatorname{Inn}(Q, \cdot)=\left\langle R_{x} R_{z T_{x}} R_{z x}^{-1}, T_{x} \mid x, z \in Q\right\rangle$.

3. $R_{(y, x)}=L_{(y, x)}$ if and only if $R_{x} R_{y T_{x}} R_{y x}^{-1}=L_{x} L_{y T_{x}^{-1}} L_{x y}^{-1}$ for all $x, y \in Q$.

Proof. 1 and 2 follow by 3 of Theorem 2.3. For 3, in a Basarab loop $(Q, \cdot), R_{(z, x)}=R_{x} R_{z T_{x}} R_{z x}^{-1}, \forall x, z \in Q$ and $L_{(y, x)}=L_{x} L_{y T_{x}^{-1}} L_{x y}^{-1}, \forall x, y \in$ $Q$. This implies, $R_{(y, x)}=R_{x} R_{y T_{x}} R_{y x}^{-1}, \forall x, y, z \in Q$. Thus, $R_{(y, x)}=$ $L_{(y, x)} \Leftrightarrow R_{x} R_{y T_{x}} R_{y x}^{-1}=L_{x} L_{y T_{x}^{-1}} L_{x y}^{-1} \quad \forall x, y \in Q$.

Theorem 2.5. In a Basarab loop $(Q, \cdot)$ the following are equivalent for all $x, y \in Q$ :

(i) $T_{x y}=T_{x} T_{y}$.

(ii) $L_{(x, y)} \in \mathcal{P}(Q, \cdot)$.

(iii) $R_{(x, y)} \in \Lambda(Q, \cdot)$.

Proof. Let $(Q, \cdot)$ be a Basarab loop and let $B_{1}(x)=\left(T_{x}^{-1}, L_{x}, L_{x}\right) \in$ $\operatorname{AUT}(Q, \cdot)$,

$$
\begin{aligned}
& B_{1}(y)=\left(T_{y}^{-1}, L_{y}, L_{y}\right) \in A U T(Q, \cdot), \text { and } B_{1}(y x)^{-1}=\left(T_{y x}, L_{y x}^{-1}, L_{y x}^{-1}\right) \\
& \qquad \in A U T(Q, \cdot) \\
& \text { then } B_{1}(x) B_{1}(y) B_{1}(y x)^{-1} \in A U T(Q, \cdot) \Rightarrow T_{x}^{-1} T_{y}^{-1} T_{y x}=I \\
& \Leftrightarrow T_{y x}=T_{y} T_{x} \Leftrightarrow\left(I, L_{(y, x)}, L_{(y, x)}\right) \in A U T(Q, \cdot) \Leftrightarrow L_{(y, x)} \in \mathcal{P}(Q, \cdot),
\end{aligned}
$$




$$
\forall x, y \in Q .
$$

Also, let $B_{2}(x)=\left(R_{x}, T_{x}, R_{x}\right) \in A U T(Q, \cdot), B_{2}(y)=\left(R_{y}, T_{y}, R_{y}\right) \in \operatorname{AUT}(Q, \cdot)$

$$
\begin{gathered}
\text { and } B_{2}(x y)^{-1}=\left(R_{x y}^{-1}, T_{x y}^{-1}, R_{x y}^{-1}\right) \in \operatorname{AUT}(Q, \cdot) \\
\text { then } B_{2}(x) B_{2}(y) B_{2}(x y)^{-1} \in \operatorname{AUT}(Q, \cdot) \Rightarrow T_{x} T_{y} T_{x y}^{-1}=I \\
\Leftrightarrow T_{x y}=T_{x} T_{y} \Leftrightarrow(R(x, y), I, R(x, y) \in A U T(Q, \cdot) \\
\Leftrightarrow R(x, y) \in \Lambda(Q, \cdot) \forall x, y \in Q .
\end{gathered}
$$

\subsection{Relationship between Basarab loop and automorphic loop}

Theorem 2.6. Let $(Q, \cdot)$ be a Basarab loop. Then:

(i) $(Q, \cdot)$ is an $A_{\lambda}$-loop if and only if $L_{(x, y)}=T_{x}^{-1} T_{y}^{-1} T_{y x}$ for all $x, y \in Q$ if and only if $R_{y x}=T_{y} R_{x} L_{y}$ if and only if $\operatorname{Inn}_{\lambda}(Q, \cdot)=\operatorname{Inn}_{\mu}(Q, \cdot)$.

(ii) $(Q, \cdot)$ is an $A_{\rho}$-loop if and only if $R_{(x, y)}=T_{x} T_{y} T_{x y}^{-1}$ for all $x, y \in Q$ if and only if $L_{x y}=T_{y}^{-1} L_{x} R_{y}$ if and only if $\operatorname{Inn}_{\rho}(Q, \cdot)=\operatorname{Inn}_{\mu}(Q, \cdot)$.

(iii) $(Q, \cdot)$ is an A-loop if and only if $\operatorname{Inn}(Q, \cdot)=\left\langle T_{x}: x \in Q\right\rangle \leq$ $A U M(Q, \cdot) \Leftrightarrow R_{y x} L_{y}^{-1} R_{x}^{-1}=T_{y}=L_{x} R_{y} L_{x y}^{-1} \in A U M(Q, \cdot)$ for all $x, y \in Q$ if and only if $\operatorname{Inn}(Q, \cdot)=\operatorname{Inn}_{\mu}(Q, \cdot) \leq A U M(Q, \cdot)$.

\section{Proof.}

(i) From the Basarab laws,

let $B_{1}(x)=\left(T_{x}^{-1}, L_{x}, L_{x}\right) \in A U T(Q, \cdot), B_{1}(y)=\left(T_{y}^{-1}, L_{y}, L_{y}\right) \in$ $A U T(Q, \cdot)$, and $B_{1}(y x)^{-1}=\left(T_{y x}, L_{y x}^{-1}, L_{y x}^{-1}\right) \in A U T(Q, \cdot)$

It follows that, $B_{1}(x) B_{1}(y) B_{1}(y x)^{-1}=\left(T_{x}^{-1} T_{y}^{-1} T_{y x}, L_{(x, y)}, L_{(x, y)}\right) \in$ $A U T(Q, \cdot)$. Then, a Basarab loop $(Q, \cdot)$ is an $A_{\lambda}$-loop $\Leftrightarrow L_{(x, y)}=$ $T_{x}^{-1} T_{y}^{-1} T_{y x}, \forall x, y \in Q . L_{(x, y)}=T_{x}^{-1} T_{y}^{-1} T_{y x} \Leftrightarrow L_{x} L_{y} L_{y x}^{-1}$ $=T_{x}^{-1} T_{y}^{-1} T_{y x} \Leftrightarrow L_{x} R_{x}^{-1} T_{y}^{-1} R_{y x} L_{y x}^{-1}=L_{x} L_{y} L_{y x}^{-1} \Leftrightarrow R_{x}^{-1} T_{y}^{-1} R_{y x}=$ $L_{y} \Leftrightarrow T_{y} R_{x} L_{y}=R_{y x}$.

(ii) Also, from the Basarab laws, let $B_{2}(x)=\left(R_{x}, T_{x}, R_{x}\right) \in A U T(Q, \cdot)$,

$B_{2}(y)=\left(R_{y}, T_{y}, R_{y}\right) \in \operatorname{AUT}(Q, \cdot)$, and

$B_{2}(x y)^{-1}=\left(R_{x y}^{-1}, T_{x y}^{-1}, R_{x y}^{-1}\right) \in A U T(Q, \cdot)$. Thus,

$B_{2}(x) B_{2}(y) B_{2}(x y)^{-1}$ 
$=\left(R_{(x, y)}, T_{x} T_{y} T_{x y}^{-1}, R_{(x, y)}\right) \in A U T(Q, \cdot)$. This implies, a Basarab loop $(Q, \cdot)$ is an $A_{\rho}$-loop $\Leftrightarrow R_{(x, y)}=T_{x} T_{y} T_{x y}^{-1} \cdot R_{(x, y)}=T_{x} T_{y} T_{x y}^{-1} \Leftrightarrow$ $R_{x} R_{y} R_{x y}^{-1}=T_{x} T_{y} T_{x y}^{-1} \Leftrightarrow T_{x} T_{y} L_{x y} R_{x y}^{-1}=R_{x} R_{y} R_{x y}^{-1} \Leftrightarrow T_{x} T_{y} L_{x y}=$ $R_{x} R_{y} \Leftrightarrow R_{x} L_{x}^{-1} T_{y} L_{x y}=R_{x} R_{y} \Leftrightarrow L_{x}^{-1} T_{y} L_{x y}=R_{y} \Leftrightarrow L_{x y}=T_{y}^{-1} L_{x} R_{y}$.

(iii) This follows from (i) and (ii).

Corollary 2.2. Let $(Q, \cdot)$ be a Basarab loop. Then:

1. $(Q, \cdot)$ is an $A_{\lambda}$-loop, $L_{(x, y)}=T_{x}^{-1} T_{y}^{-1} T_{y x}, R_{y x}=T_{y} R_{x} L_{y}$ and $\operatorname{Inn}_{\lambda}(Q, \cdot)$ $=\operatorname{Inn}_{\mu}(Q, \cdot)$ for all $x, y \in Q$.

2. $(Q, \cdot)$ is an $A_{\rho}$-loop, $R_{(x, y)}=T_{x} T_{y} T_{x y}^{-1}, L_{x y}=T_{y}^{-1} L_{x} R_{y}$ and $\operatorname{Inn}_{\rho}(Q, \cdot)=$ $\operatorname{Inn}_{\mu}(Q, \cdot)$ for all $x, y \in Q$.

3. $(Q, \cdot)$ is an A-loop if and only if $\operatorname{Inn}(Q, \cdot)=\left\langle T_{x}: x \in Q\right\rangle \leq$ $A U M(Q, \cdot) \Leftrightarrow T_{y}=R_{y x} L_{y}^{-1} R_{x}^{-1}=L_{x} R_{y} L_{x y}^{-1} \in \operatorname{AUM}(Q, \cdot)$ if and only if $\operatorname{Inn}(Q, \cdot)=\operatorname{Inn}_{\mu}(Q, \cdot) \leq A U M(Q, \cdot)$ for all $x, y \in Q$.

Proof. In a Basarab loop $(Q, \cdot),\left(T_{x}^{-1} T_{y}^{-1} T_{y x}, L_{(y, x)}, L_{(y, x)}\right),\left(R_{(x, y)}\right.$, $\left.T_{x} T_{y} T_{x y}^{-1}, R_{(x, y)}\right) \in A U T(Q, \cdot)$. Since $e L_{(y, x)}=e=e R_{(x, y)}$, then $L_{(y, x)}, R_{(x, y)} \in A U M(Q, \cdot)$. Other conclusions follow from Theorem 2.6.

\subsection{Basarab loop and generators of its total multiplication group}

Theorem 2.7. Let $(Q, \cdot)$ be a Basarab loop and let $U_{x}=M_{x} R_{x}^{-1}, V_{x}=$ $M_{x}^{-1} L_{x}^{-1}, W_{x}=R_{x} M_{x}$ for any arbitrarily fixed $x \in Q$.

1. $T_{x}^{-1}=J_{\rho} L_{x} M_{x}^{-1}$.

2. $T_{x}=J_{\lambda} R_{x} M_{x}$.

3. The following are true

1. $T_{x}=W_{x} V_{x}$.

2. $M_{x}^{2} V_{x}=U_{x} T_{x}$.

3. $U_{x} T_{x}^{2}=T_{x} W_{x}$.

4. $R_{x}=L_{x} \Leftrightarrow M_{x}^{2}=T_{x} J_{\rho}^{2} T_{x}$. 
5. $\left|M_{x}\right|=2 \Leftrightarrow T_{x} J_{\lambda}=J_{\rho} T_{x}^{2}$.

6. $\left|T_{x}\right|=2 \Leftrightarrow J_{\rho}^{2}=W_{x} U_{x} T_{x}$.

7. $J_{\rho} U_{x} T_{x}=W_{x} J_{\rho}$.

8. $J_{\rho} R_{x}=R_{x} T_{x} J_{\rho}$.

9. $T_{x} J_{\rho}=J_{\rho} T_{x} \Leftrightarrow U_{x} T_{x}=W_{x}$.

10. $R_{x}^{n} T_{x} J_{\rho} L_{x}^{n}=R_{x}^{n-1} J_{\rho} T_{x} L_{x}^{n-1} \forall n \in \mathbf{N}$.

11. $J_{\lambda} T_{x}=T_{x} V_{x}$.

12. $W_{x}=J_{\rho} T_{x}$.

13. $U_{x} T_{x}=T_{x} J_{\rho}$.

14. $U_{x} W_{x}=M_{x}^{2}$.

15. $W_{x} U_{x} R_{x}=R_{x} U_{x} W_{x}$.

16. $M_{x} U_{x} R_{x}=U_{x} W_{x}$.

17. $M_{x}=U_{x} R_{x}$ and $W_{x}=R_{x} M_{x}$.

4. The following are equivalent:

1. $J_{\rho}=J_{\lambda}$.

2. $T_{x}^{2}=U_{x} T_{x} R_{x} M_{x}$.

3. $T_{x}^{2}=U_{x} T_{x} W_{x}$.

4. $U_{x} T_{x}^{2}=T_{x}^{2} V_{x}$.

Hence, $\mathcal{T} \mathcal{M}(Q, \cdot)=\left\langle\left\{R_{x}, L_{x}, U_{x}\right\} \mid x \in Q\right\rangle=\left\langle\left\{R_{x}, L_{x}, V_{x}\right\}\right| x \in$ $Q\rangle=\left\langle\left\{R_{x}, L_{x}, W_{x}\right\} \mid x \in Q\right\rangle$.

5. $T_{x}^{2}=U_{x}^{n} T_{x}^{2} V_{x}^{n}$ and $T_{x}^{-2}=V_{x}^{n} T_{x}^{-2} U_{x}^{n}$ for all $n \in \mathbf{N}$. If $\left|T_{x}\right|=2$, then $U_{x}^{n} V_{x}^{n}=I$ for all $x \in \mathbf{N}$.

6. The following are true for any $n \in \mathbf{N}$ :

1. $J_{\rho}^{n}=T_{x}^{-1} U_{x}^{n} T_{x}$.

2. $\left|J_{\rho}\right|=n \Leftrightarrow\left|U_{x}\right|=n$.

3. $J_{\lambda}^{n}=T_{x} V_{x}^{n-1} W_{x}^{-1}$.

4. $\left|J_{\lambda}\right|=n \Leftrightarrow T_{x} V_{x}^{n-1}=W_{x}$.

5. $J_{\rho}^{n}=J_{\lambda}^{n} \Leftrightarrow T_{x}^{2} V_{x}^{n}=U_{x}^{n} T_{x}^{2}$. 


\section{Proof.}

1. From property (ii) of Lemma $2.1, x \cdot y x^{\rho}=x /\left(x y^{\rho}\right)=\left(x y^{\rho}\right) M_{x}^{-1} \Rightarrow$ $y R_{x^{\rho}} L_{x}=y J_{\rho} L_{x} M_{x}^{-1} \Rightarrow T_{x}^{-1}=J_{\rho} L_{x} M_{x}^{-1}$.

2. From property (iv) of Lemma 2.1, $x^{\lambda} z \cdot x=z^{\lambda} x \backslash x \Rightarrow z L_{x^{\lambda}} \cdot x=$ $\left(z^{\lambda} x\right) M_{x} \Rightarrow z L_{x^{\lambda}} R_{x}=z J_{\lambda} R_{x} M_{x} \Rightarrow L_{x^{\lambda}} R_{x}=J_{\lambda} R_{x} M_{x} \Rightarrow L_{x J_{\lambda}} R_{x}=$ $J_{\lambda} R_{x} M_{x} \Rightarrow T_{x}=J_{\lambda} R_{x} M_{x}$.

3. 1. $W_{x} V_{x}=R_{x} M_{x} M_{x}^{-1} L_{x}^{-1}=T_{x} \Rightarrow T_{x}=W_{x} V_{x}$.

2. $M_{x}^{2} V_{x} T_{x}^{-1}=M_{x}^{2} M_{x}^{-1} L_{x}^{-1} L_{x} R_{x}^{-1}=M_{x} R_{x}^{-1}=U_{x} \Rightarrow M_{x}^{2} V_{x}=$ $U_{x} T_{x}$.

3. From 1 and 2: $J_{\lambda}=T_{x} M_{x}^{-1} R_{x}^{-1}$ and $J_{\lambda}=L_{x} M_{x}^{-1} T_{x}$. So, $T_{x} M_{x}^{-1} R_{x}^{-1}=L_{x} M_{x}^{-1} T_{x} \Rightarrow T_{x}\left(R_{x} M_{x}\right)^{-1}=L_{x} M_{x}^{-1} T_{x} \Rightarrow T_{x} W_{x}^{-1}=$ $L_{x} M_{x} M_{x}^{-2} T_{x}=V_{x}^{-1} M_{x}^{-2} T_{x} \Rightarrow T_{x} W_{x}^{-1}=V_{x}^{-1} M_{x}^{-2} T_{x} \Rightarrow V_{x} T_{x}=$ $M_{x}^{-2} T_{x} W_{x} \Rightarrow M_{x}^{2} V_{x} T_{x}=T_{x} W_{x} \Rightarrow U_{x} T_{x}^{2}=T_{x} W_{x}$.

4. From 1 and 2: $L_{x}=J_{\lambda} T_{x}^{-1} M_{x}$ and $R_{x}=J_{\rho} T_{x} M_{x}^{-1}$. So, $L_{x}=$ $R_{x} \Leftrightarrow J_{\lambda} T_{x}^{-1} M_{x}=J_{\rho} T_{x} M_{x}^{-1} \Leftrightarrow M_{x}^{2}=T_{x} J_{\rho}^{2} T_{x}$. Thus, $R_{x}=$ $L_{x} \Leftrightarrow M_{x}^{2}=T_{x} J_{\rho}^{2} T_{x}$.

5. From 1 and 2: $M_{x}=R_{x}^{-1} J_{\rho} T_{x}$ and $M_{x}^{-1}=L_{x}^{-1} J_{\lambda} T_{x}^{-1}$. So, $\left|M_{x}\right|=2 \Leftrightarrow M_{x}^{-1}=M_{x} \Leftrightarrow R_{x}^{-1} J_{\rho} T_{x}=L_{x}^{-1} J_{\lambda} T_{x}^{-1} \Leftrightarrow J_{\rho} T_{x}^{2}=$ $T_{x} J_{\lambda}$. Thus, $\left|M_{x}\right|=2 \Leftrightarrow T_{x} J_{\lambda}=J_{\rho} T_{x}^{2}$.

6. From 1 and 2: $\left|T_{x}\right|=2 \Leftrightarrow T_{x}^{-1}=T_{x} \Leftrightarrow J_{\rho} L_{x} M_{x}^{-1}=J_{\lambda} R_{x} M_{x} \Leftrightarrow$ $J_{\rho}^{2}=R_{x} M_{x} M_{x} L_{x}^{-1}=W_{x} M_{x} L_{x}^{-1}=W_{x} M_{x}^{2} M_{x}^{-1} L_{x}^{-1}=W_{x} M_{x}^{2} V_{x}=$ $W_{x} U_{x} T_{x} \Leftrightarrow J_{\rho}^{2}=W_{x} U_{x} T_{x}$. Thus, $\left|T_{x}\right|=2 \Leftrightarrow J_{\rho}^{2}=W_{x} U_{x} T_{x}$.

7. From 1 and 2: $I=T_{x} T_{x}^{-1}=J_{\lambda} R_{x} M_{x} J_{\rho} L_{x} M_{x}^{-1} \Rightarrow R_{x}^{-1} J_{\rho} M_{x}=$ $M_{x} J_{\rho} L_{x} \Rightarrow J_{\rho} M_{x} L_{x}^{-1}=R_{x} M_{x} J_{\rho} \Rightarrow J_{\rho} M_{x}^{2} M_{x}^{-1} L_{x}^{-1}=W_{x} J_{\rho} \Rightarrow$ $J_{\rho} M_{x}^{2} V_{x}=W_{x} J_{\rho} \Rightarrow J_{\rho} U_{x} T_{x}=W_{x} J_{\rho}$.

8. From 1 and 2: $M_{x}=R_{x}^{-1} J_{\rho} T_{x}$ and $M_{x}=T_{x} J_{\rho} L_{x}$. So, $R_{x}^{-1} J_{\rho} T_{x}=$ $T_{x} J_{\rho} L_{x} \Rightarrow J_{\rho} R_{x}=R_{x} T_{x} J_{\rho}$.

9. From 1 and 2: $T_{x}^{-1} J_{\lambda}=M_{x}^{-1} R_{x}^{-1}$ and $J_{\lambda} T_{x}^{-1}=L_{x} M_{x}^{-1}$. So, $T_{x} J_{\rho}=J_{\rho} T_{x} \Leftrightarrow M_{x}^{-1} R_{x}^{-1}=L_{x} M_{x}^{-1} \Leftrightarrow\left(R_{x} M_{x}\right)^{-1}=L_{x} M_{x} M_{x}^{-2} \Leftrightarrow$ $W_{x}^{-1}=V_{x}^{-1} M_{x}^{-2} \Leftrightarrow U_{x} T_{x}=W_{x}$. Thus, $T_{x} J_{\rho}=J_{\rho} T_{x} \Leftrightarrow U_{x} T_{x}=$ $W_{x}$.

10. From 1 and 2: $M_{x}=R_{x}^{-1} J_{\rho} T_{x}$ and $M_{x}^{-1}=L_{x}^{-1} J_{\lambda} T_{x}^{-1}$. So, $I=M_{x} M_{x}^{-1}=R_{x}^{-1} J_{\rho} T_{x} L_{x}^{-1} J_{\lambda} T_{x}^{-1} \Rightarrow R_{x} T_{x} J_{\rho}=J_{\rho} T_{x} L_{x}^{-1} \Rightarrow$ $R_{x} T_{x} J_{\rho} L_{x}=J_{\rho} T_{x}$. So, $R_{x}^{n} T_{x} J_{\rho} L_{x}^{n}=R_{x}^{n-1} J_{\rho} T_{x} L_{x}^{n-1} \forall n \in \mathbf{N}$. 
11. From $2: M_{x}^{-1}=T_{x}^{-1} J_{\lambda} R_{x}$. So, $M_{x}^{-1} L_{x}^{-1}=V_{x}=T_{x}^{-1} J_{\lambda} R_{x} L_{x}^{-1}=$ $T_{x}^{-1} J_{\lambda} T_{x} \Rightarrow V_{x} T_{x}^{-1}=T_{x}^{-1} J_{\lambda} \Rightarrow J_{\lambda} T_{x}=T_{x} V_{x}$.

12. From 2: $M_{x}=R_{x}^{-1} J_{\rho} T_{x}$. So, $W_{x}=R_{x} M_{x}=R_{x} R_{x}^{-1} J_{\rho} T_{x}=$ $J_{\rho} T_{x} \Rightarrow W_{x}=J_{\rho} T_{x}$.

13. From 1: $M_{x}=T_{x} J_{\rho} L_{x}$. Now, $U_{x}=M_{x} R_{x}^{-1}=T_{x} J_{\rho} L_{x} R_{x}^{-1}=$ $T_{x} J_{\rho} T_{x}^{-1} \Rightarrow U_{x} T_{x}=T_{x} J_{\rho}$.

14. $U_{x} W_{x}=M_{x} R_{x}^{-1} R_{x} M_{x}=M_{x}^{2} \Rightarrow U_{x} W_{x}=M_{x}^{2}$.

15. $W_{x} U_{x}=R_{x} M_{x}^{2} R_{x}^{-1} \Rightarrow W_{x} U_{x} R_{x}=R_{x} M_{x}^{2}=R_{x} U_{x} W_{x} \Rightarrow$ $W_{x} U_{x} R_{x}=R_{x} U_{x} W_{x}$.

16. $M_{x} U_{x}=M_{x}^{2} R_{x}^{-1}=U_{x} W_{x} R_{x}^{-1} \Rightarrow M_{x} U_{x} R_{x}=U_{x} W_{x}$.

17. Trivial.

4. We shall use 1 and 2 .

(a) $\Leftrightarrow$ (b) $J_{\lambda}=J_{\rho} \Leftrightarrow T_{x} M_{x}^{-1} R_{x}^{-1}=T_{x}^{-1} M_{x} L_{x}^{-1} \Rightarrow T_{x} M_{x}^{-1} R_{x}^{-1} R_{x}=$ $T_{x}^{-1} M_{x} L_{x}^{-1} R_{x} \Rightarrow T_{x}^{2} M_{x}^{-1}=M_{x} L_{x}^{-1} R_{x}=M_{x} R_{x}^{-1} R_{x} L_{x}^{-1} R_{x} \Rightarrow$ $T_{x}^{2} M_{x}^{-1}=U_{x} T_{x} R_{x} \Rightarrow T_{x}^{2}=U_{x} T_{x} R_{x} M_{x}$.

(a) $\Leftrightarrow$ (c) $J_{\lambda}=J_{\rho} \Leftrightarrow T_{x}^{2}=U_{x} T_{x} R_{x} M_{x} \Leftrightarrow T_{x}^{2}=U_{x} T_{x} W_{x}$.

(a) $\Leftrightarrow$ (d) $J_{\lambda}=J_{\rho} \Leftrightarrow T_{x}^{2}=U_{x} T_{x} R_{x} M_{x} \Leftrightarrow U_{x}=T_{x}^{2}\left(T_{x} R_{x} M_{x}\right)^{-1} \Leftrightarrow$ $U_{x}=T_{x}^{2} M_{x}^{-1} R_{x}^{-1} T_{x}^{-1} \Leftrightarrow U_{x}=T_{x}^{2} M_{x}^{-1} L_{x}^{-1} L_{x} R_{x}^{-1} T_{x}^{-1} \Leftrightarrow U_{x}=$ $T_{x}^{2} V_{x} T_{x}^{-2} \Leftrightarrow U_{x} T_{x}^{2}=T_{x}^{2} V_{x}$.

$\mathcal{T} \mathcal{M}(Q, \cdot)=\left\langle\left\{R_{x}, L_{x}, U_{x}\right\} \mid x \in Q\right\rangle=\left\langle\left\{R_{x}, L_{x}, V_{x}\right\} \mid x \in Q\right\rangle=$ $\left\langle\left\{R_{x}, L_{x}, W_{x}\right\} \mid x \in Q\right\rangle$ follows from the fact that $T_{x}^{2}=U_{x} T_{x} R_{x} M_{x} \Leftrightarrow$ $M_{x}=\left(U_{x} T_{x} R_{x}\right)^{-1} T_{x}^{2}$.

5. From 1 and $2, J_{\rho}=T_{x}^{-1} M_{x} L_{x}^{-1}$ and $J_{\lambda}=T_{x} M_{x}^{-1} R_{x}^{-1}$. Hence:

$$
\begin{gathered}
T_{x}^{-1} M_{x} L_{x}^{-1} T_{x} M_{x}^{-1} R_{x}^{-1}=I \text { and } T_{x} M_{x}^{-1} R_{x}^{-1} T_{x}^{-1} M_{x} L_{x}^{-1}=I \Rightarrow \\
T_{x}^{-1} M_{x} R_{x}^{-1} R_{x} L_{x}^{-1} T_{x} M_{x}^{-1} L_{x}^{-1} L_{x} R_{x}^{-1}=I \text { and } \\
T_{x} M_{x}^{-1} L_{x}^{-1} L_{x} R_{x}^{-1} T_{x}^{-1} M_{x} R_{x}^{-1} R_{x} L_{x}^{-1}=I \Rightarrow \\
T_{x}^{-1} U_{x} T_{x}^{2} V_{x} T_{x}^{-1}=I \text { and } T_{x} V_{x} T_{x}^{-2} U_{x} T_{x}=I \Rightarrow \\
\underbrace{U_{x} T_{x}^{2} V_{x}=T_{x}^{2}}_{a} \text { and } \underbrace{V_{x} T_{x}^{-2} U_{x}=T_{x}^{-2}}_{b}
\end{gathered}
$$

By multiplying (2.1)(a) and (2.1)(b), we get 


$$
U_{x}^{2} T_{x}^{2} V_{x}^{2}=T_{x}^{2}
$$

Substituting eq:bs2 in (2.1)(a), we get $U_{x}^{3} T_{x}^{2} V_{x}^{3}=T_{x}^{2}$. Making this substitution inductively, we have $T_{x}^{2}=U_{x}^{n} T_{x}^{2} V_{x}^{n}$ for all $\mathbf{N}$. Doing a similar thing with eq:bs2 and (2.1)(b), we get $T_{x}^{-2}=V_{x}^{n} T_{x}^{-2} U_{x}^{n}$ for all $\mathbf{N}$.

6. We shall use 1, 2 and some results in 3 .

(a) $J_{\rho}^{2}=T_{x}^{-1} M_{x} L_{x}^{-1} T_{x}^{-1} M_{x} L_{x}^{-1}=T_{x}^{-1} M_{x} L_{x}^{-1} L_{x} R_{x}^{-1} M_{x} L_{x}^{-1}$ $=T_{x}^{-1} M_{x} R_{x}^{-1} M_{x} L_{x}^{-1}=T_{x}^{-1} U_{x} M_{x}^{2} M_{x}^{-1} L_{x}^{-1}=T_{x}^{-1} U_{x} M_{x}^{2} V_{x}=$ $T_{x}^{-1} U_{x}^{2} T_{x}$. Hence, $J_{\rho}^{3}=J_{\rho}^{2} J_{\rho}=T_{x}^{-1} U_{x}^{2} T_{x} T_{x}^{-1} U_{x} T_{x}=T_{x}^{-1} U_{x}^{3} T_{x}$. Continuing by induction, we have $J_{\rho}^{n}=T_{x}^{-1} U_{x}^{n} T_{x}$.

(b) This follows from (a).

(c) $J_{\lambda}^{2}=T_{x} M_{x}^{-1} R_{x}^{-1} T_{x} M_{x}^{-1} R_{x}^{-1}=T_{x} M_{x}^{-1} R_{x}^{-1} R_{x} L_{x}^{-1} M_{x}^{-1} R_{x}^{-1}=$ $T_{x} M_{x}^{-1} L_{x}^{-1}\left(R_{x} M_{x}\right)^{-1} \Rightarrow J_{\lambda}^{2}=T_{x} V_{x} W_{x}^{-1}$. Hence, $J_{\lambda}^{3}=J_{\lambda}^{2} J_{\lambda}=$ $T_{x} V_{x} W_{x}^{-1} T_{x} W_{x}^{-1}=T_{x} V_{x}^{2} W_{x}^{-1}$. Continuing by induction, we have $J_{\lambda}^{n}=T_{x} V_{x}^{n-1} W_{x}^{-1}$.

(d) This follows from (c).

(e) (a) and (c) answer this.

Lemma 2.3. Let $(Q, \cdot)$ be a Basarab loop. The following are equivalent:

1. $(Q, \cdot)$ is a cross inverse property loop.

2. $(Q, \cdot)$ is commutative.

3. $(Q, \cdot)$ is an abelian group.

4. $L_{x} \in \mathcal{P}(Q, \cdot)$ for all $x \in Q$.

5. $R_{x} \in \Lambda(Q, \cdot)$ for all $x \in Q$.

Proof. By Lemma 2.1, $x \cdot y=\left(x \cdot y x^{\rho}\right) \cdot x$. If $(Q, \cdot)$ has the CIP, then $x \cdot y=\left(x \cdot y x^{\rho}\right) \cdot x \Rightarrow x \cdot y=y \cdot x$ which implies commutativity. The converse is also true. By BK1 of $(1.2),\left(x \cdot y x^{\rho}\right)(x z)=x \cdot y z .(Q, \cdot)$ has CIP if and only if $y \cdot x z=x \cdot y z \Leftrightarrow(Q, \cdot)$ is an abelian group. By Lemma 2.2, $\left(T_{x}^{-1}, L_{x}, L_{x}\right),\left(R_{x}, T_{x}, R_{x}\right) \in \operatorname{AUT}(Q, \cdot) .\left(T_{x}^{-1}, L_{x}, L_{x}\right) \in$ 
$\operatorname{AUT}(Q, \cdot) \Rightarrow\left(L_{x} R_{x}^{-1}, L_{x}, L_{x}\right) \in \operatorname{AUT}(Q, \cdot)$. So, $(Q, \cdot)$ is commutative if and only if $L_{x} \in \mathcal{P}(Q, \cdot)$ for all $x \in Q .\left(R_{x}, T_{x}, R_{x}\right) \in \operatorname{AUT}(Q, \cdot) \Rightarrow$ $\left(R_{x}, R_{x} L_{x}^{-1}, R_{x}\right) \in \operatorname{AUT}(Q, \cdot)$. So, $(Q, \cdot)$ is commutative if and only if $R_{x} \in \Lambda(Q, \cdot)$ for all $x \in Q$.

Lemma 2.4. A Basarab loop is a Moufang loop if and only if it is flexible.

Proof. By Lemma 2.2, $\left(T_{x}^{-1}, L_{x}, L_{x}\right),\left(R_{x}, T_{x}, R_{x}\right) \in \operatorname{AUT}(Q, \cdot)$. $\left(T_{x}^{-1}, L_{x}, L_{x}\right)\left(R_{x}, T_{x}, R_{x}\right)=\left(T_{x}^{-1} R_{x}, L_{x} T_{x}, L_{x} R_{x}\right)=\left(L_{x}, L_{x} R_{x} L_{x}^{-1}, L_{x} R_{x}\right) \in$ $\operatorname{AUT}(Q, \cdot)$. So, the loop is flexible if and only if $L_{x} R_{x}=R_{x} L_{x} \Leftrightarrow\left(L_{x}, R_{x}, L_{x} R_{x}\right) \in$ $A U T(Q, \cdot) \Leftrightarrow(x y)(z x)=(x \cdot y z) x$ if and only if the loop is a Moufang loop.

Theorem 2.8. A Basarab loop is a TA-loop if and only if it is a commutative and flexible loop.

Proof. This follows from Lemma 2.4 and Theorem 1.1.

Corollary 2.3. A Basarab loop $(Q, \cdot)$ is a TA-loop if and only if it is a flexible loop and any of the following is true.

1. $(Q, \cdot)$ is a cross inverse property loop.

2. $(Q, \cdot)$ is commutative.

3. $(Q, \cdot)$ is an abelian group.

4. $L_{x} \in \mathcal{P}(Q, \cdot)$ for all $x \in Q$.

5. $R_{x} \in \Lambda(Q, \cdot)$ for all $x \in Q$.

Proof. This follows from Lemma 2.3 and Theorem 2.8.

Theorem 2.9. Let $(Q, \cdot)$ be a Basarab loop. Then:

$$
\begin{gathered}
\mathcal{T M}(Q, \cdot)=\left\langle\left\{R_{x}, L_{x}, R_{x}^{-1} J_{\rho} T_{x}: x \in Q\right\}\right\rangle \\
=\left\langle\left\{R_{x}, L_{x}, T_{x} J_{\rho} L_{x}: x \in Q\right\}\right\rangle \text { and } \\
\operatorname{TInn}(Q, \cdot) \\
=\left\langle\left\{T_{x}, T_{x} T_{y} T_{x y}^{-1}, T_{x}^{-1} T_{y}^{-1} T_{y x}, T_{y} J_{\rho} L_{y} R_{x}^{-1} J_{\rho} T_{x} T_{y \backslash x}^{-1} J_{\lambda} R_{y \backslash x}, T_{x} J_{\rho} T_{x}^{-1} \mid x, y \in Q\right\}\right\rangle \\
=\left\langle\left\{T_{x}, T_{x} T_{y} T_{x y}^{-1}, T_{x}^{-1} T_{y}^{-1} T_{y x}, T_{y} J_{\rho} L_{y} R_{x}^{-1} J_{\rho} T_{x} L_{y \backslash x}^{-1} J_{\lambda} T_{y \backslash x}^{-1}, T_{x} J_{\rho} T_{x}^{-1} \mid x, y \in Q\right\}\right\rangle .
\end{gathered}
$$


Proof. This is proved by Corollary 2.2 and Theorem 2.7. Recall that $\mathcal{T} \mathcal{M}(Q, \cdot)=\left\langle\left\{R_{x}, L_{x}, M_{x}: x \in Q\right\}\right\rangle$ and $\operatorname{TInn}(Q, \cdot)=\left\langle R_{(x, y)}, L_{(x, y)}, T_{x}, M_{(x, y)}, U_{x} \mid x, y \in Q\right\rangle$ where $M_{(x, y)}=$ $M_{y} M_{x} M_{y \backslash x}^{-1}$ and $U_{x}=M_{x} R_{x}^{-1}$.

$U_{x}=T_{x} J_{\rho} T_{x}^{-1}$ and $M_{(x, y)}=M_{y} M_{x} M_{y \backslash x}^{-1}$ while

$M_{(x, y)}=T_{y} J_{\rho} L_{y} R_{x}^{-1} J_{\rho} T_{x}\left(T_{y \backslash x} J_{\rho} L_{y \backslash x}\right)^{-1}=T_{y} J_{\rho} L_{y} R_{x}^{-1} J_{\rho} T_{x} L_{y \backslash x}^{-1} J_{\lambda} T_{y \backslash x}^{-1}$ and

$M_{(x, y)}=T_{y} J_{\rho} L_{y} R_{x}^{-1} J_{\rho} T_{x}\left(R_{y \backslash x}^{-1} J_{\rho} T_{y \backslash x}\right)^{-1}=T_{y} J_{\rho} L_{y} R_{x}^{-1} J_{\rho} T_{x} T_{y \backslash x}^{-1} J_{\lambda} R_{y \backslash x}$.

Remark 2.1. Theorem 2.9 gives expressions for the total multiplication group and total inner mapping group of a Basarab loop in terms of finetuned generators. Hence, it is an ostensible solution to Question 1.2.

Theorem 2.10. 1. Let $(Q, \cdot)$ be a Basarab loop. The following are equivalent.

1. $\left\langle\left\{L_{x \backslash y}^{-1} T_{y} J_{\rho} L_{y} R_{x}^{-1} J_{\rho} T_{x}, R_{y / x}^{-1} T_{y}^{-1} J_{\lambda} R_{y} L_{x}^{-1} J_{\lambda} T_{x}^{-1} \mid x, y \in Q\right\}\right\rangle \leq$ $A U M(Q, \cdot)$.

2. $\left\langle T_{x}: x \in Q\right\rangle \leq A U M(Q, \cdot)$ and $(Q, \cdot)$ is flexible.

Proof. This follows by Theorem 1.2, Theorem 2.6 and Theorem 2.7.

$M_{y} M_{x}=T_{y} J_{\rho} L_{y} R_{x}^{-1} J_{\rho} T_{x}$ and $M_{y}^{-1} M_{x}^{-1}=\left(M_{x} M_{y}\right)^{-1}=\left(T_{x} J_{\rho} L_{x} R_{y}^{-1} J_{\rho} T_{y}\right)^{-1}$ $=T_{y}^{-1} J_{\lambda} R_{y} L_{x}^{-1} J_{\lambda} T_{x}^{-1}$.

Remark 2.2. Theorem 2.10 shows that a class of total inner mappings acts on a Basarab loop $Q$ by automorphisms if and only if $Q$ is an Aloop and flexible. Theorem 2.8 and Theorem 2.10 give partial answers to Question 1.1 in the case of Basarab loop.

\section{References}

[1] A. S. Basarab, "Non-associative extensions of groups by means of abelian groups," in Proceedings of the International Conference on Group Theory: Timișoara, 17-20 September, 1992, 1993, pp. 6-10. 
[2] A. S. Basarab, "K-loops", Buletinul Academiei de Ştiinţe a Republicii Moldova. Matematica, no. 1, pp. 28-33, 1992.

[3] A. S. Basarab, "Generalized Moufang G-loops", Quasigroups and related systems, vol. 3, pp. 1-5, 1996. [Online]. Available: https:/ / bit.ly/ 3eEyDZs

[4] A. S. Basarab, "IK-loops", Quasigroups and related system, vol. 4, pp. 1-7, 1997. [Online]. Available: https:/ / bit.ly/ 3eFQqiT

[5] V. D. Belousov, Foundations of the theory of quasigroups and loops. Moscow: Nauka, 1967.

[6] V. D. Belousov, "The group associated with a quasigroup", Matematicheskie issledovaniya, vol. 4, pp. 21-39, 1969.

[7] R. H. Bruck, A survey of binary systems. Berlin: Springer, 1966.

[8] O. Chein, H. O. Pflugfelder, and J. D. H. Smith, Quasigroups and loops: theory and applications. Berlin: Heldermann, 1990.

[9] J. Dene and A. D. Keedwell, Latin squares and their applications. London: English University Press, 1974

[10] G. O. Effiong, T. G. Jaiyéọlá, and M. C. Obi, “Connections between Basarab and Buchsteiner loops", Submitted.

[11] G. O. Effiong, T. G. Jaiyéọlá, M. C. Obi, and L. S. Akinola, "Holomorphy of Basarab Loops", Submitted.

[12] E. G. Goodaire, E. Jespers, and C. P. Milies, Alternative loop rings. Amsterdam: Elsevier, 1996.

[13] I. Grecu and P. Syrbu, "On some isostrophy invariants of Bol loops", Bulletin of the Transilvania University of Braşov. Series III, mathematics, informatics, physics, vol. 5 (Special Issue), pp. 145-154, 2012. [Online]. Available: https:/ / bit.ly/ 2V2f726

[14] H. Kiechle, Theory of K-loops. Berlin: Springer, 2002, doi: 10.1007/ b83276

[15] T. G. Jaiyéọlá, A study of new concepts in Smarandache quasigroups and loops. Ann Arbor, MI: ProQuest Information and Learning, 2009. 
[16] T. G. Jaiyéọlá and J. O. Adéníran, “On the existence of A-loops with some commutative inner mappings and others of order 2", South East Asian bulletin of mathematics, vol. 33, no. 5, pp. 853-864, 2009. [Online]. Available: https:/ / bit.ly/ 3xVke2B

[17] T. G. Jaiyéọlá and G. O. Effiong, "Basarab loop and its variance with inverse properties", Quasigroups and related systems, vol. 26, no. 2, pp. 229-238, 2018. [Online]. Available: https:/ / bit.ly/ 3Buks2Q

[18] M. K. Kinyon, "Totally automorphic loops," in The Third Mile High Conference on Nonassociative Mathematics, University of Denver, Denver, Colorado, USA, August 11-17, 2013, 2013 [Online]. Available: https:/ / bit.ly/ 3rnXw0A

[19] M. K. Kinyon, K. Kunen, J. D. Phillips, and P. Vojtěchovský, "The structure of automorphic loops", Transactions of the American Mathematical Society, vol. 368, no. 12, pp. 8901-8927, 2016, doi: 10.1090/ tran/ 6622

[20] M. L. Merlini Giuliani and G. Souza dos Anjos, "On the structure of the automorphism group of certain automorphic loop", Communications in algebra, vol. 45, no. 5, pp. 2245-2255, 2017, doi: 10.1080/00927872. 2016.1233194

[21] H. O. Pflugfelder, Quasigroups and loops: introduction. Berlin: Heldermann, 1990.

[22] V. A. Shcherbakov, "Some properties of the full associated group of an IP-loop", Izvestya Akademii Nauk Moldavskoi SSR. Seria FizikoTekhnicheskikh i Matematicheskikh Nauk, vol. 79, no. 2, pp. 51- 52, 1984.

[23] J. D. H. Smith, An introduction to quasigroups and their representations. Boca Raton, FL: Chapman and Hall/ CRC, 2007.

[24] L. R. Soikis, "The special loops," in Questions of the theory of quasigroups and loops, V. D. Belousov, Ed. Kishinev: Akademii Nauk Moldavskoi SSR, 1970, pp. 122-131.

[25] D. Stanovský and P. Vojtěchovský, "Commutator theory for loops", Journal of algebra, vol. 399, pp. 290-322, 2014, doi: 10.1016/j.jalgebra.2013.08.045

[26] A. A. Ungar, "The relativistic noncommutative nonassociative group of velocities and the Thomas rotation", Results in mathematics, vol. 16, pp. 168-1789, 1989, doi: 10.1007/ BF03322653

[27] P. Syrbu, "On middle Bol loops", ROMAI Journal, vol. 6, no. 2, pp. 229-236, 2010. 
[28] P. Syrbu, "On a generalization of the inner mapping group", in Proceedings of the Fourth Conference of Mathematical Society of Moldova, dedicated to the centenary of Vladimir Andrunachievici (1917-1997) June 28 - July 2, Chisinau, 2017, 2017, pp. 161-164. [Online]. Available: https:/ / bit.ly/ 3y1kywU

[29] P. Syrbu and I. Grecu, "On some groups related to middle Bol loops", Studia Universitatis Moldaviae: Ştiinţe Exacte şi Economice (online), no. 7, pp. 10-18, 2013. [Online]. Available: https:/ / bit.ly/ 3Bqaakv

[30] P. Syrbu and I. Grecu, "On total inner mapping groups of middle Bol loops", in Proceedings of the The Fifth Conference of Mathematical Society of the Republic of Moldova dedicated to the 55th anniversary of the foundation of Vladimir Andrunachievici Institute of Mathematics and Computer Science September 28 - October 1, Chisinau, 2019, 2019, pp. 161-164. [Online]. Available: https:/ / bit.ly/ 3iyhTEp

[31] W. B. Vasantha Kandasamy, "Smarandache loops", 2002, arXiv:math/ 0307028.

[32] P. Vojtěchovský, "Three lectures on automorphic loops", Quasigroups related systems, vol. 23, no. 1, pp. 129-163, 2015. [Online]. Available: https:/ / bit.ly/ 3xVFlSs 